Gillespie and Blagrove, A ust. J. Plant Physiol., 2, 29-39; 1975). They have found that in lupins the relative proportions of the major seed proteins can be changed up to a point by supplying sulphur at different levels; in highsulphur treatments the proportion of a high-sulphur protein was increased. Thus, there is a strong gene-environmental interaction, although whether the effect is directly on gene expression at the transcription or translation levels, or whether it operates by a changed physiology, is not clear. It will be of great interest to see how general these results are for other legumes.

T. Behre (Debre Zeit, Ethiopia) reported a breakthrough on breeding Eragrostis tef, which represents a significant source of protein in Ethiopia. Up to now, all attempts at controlling the pollination of this crop have been unsuccessful. But as a result of studying the basic reproductive process of the plant, it has been possible to perform hand pollinations, so that normal procedures of plant breeding may be applied and it should now be possible to make improvements in yield through breeding.

In the realm of improved techniques for screening and evaluating the nutritional quality of advanced lines, there was an encouraging report concerning the use of a fluorescence technique for the specific determination of lysine in ground samples of grain. Less advanced is some work on a similar technique for tryptophan determination. Both of these developments were reported by A. K. Kaul (Institut fur Strahlenbotanik, Hannover).

A number of contractors from developing countries reported progress on the development of improved protein genotypes in several crops, including bread and durum wheat, rice, barley and several legumes. Because of the early stage of the work, no attempt is made to detail the results here, but a general feeling of optimism prevailed. For example, H. K. Jain (New Delhi) was convinced that tropical legumes as a group were excellent materials in which great improvements could be achieved.

\section{A homogeneous Universe?}

from Malcolm MacCallum

THE simplest cosmological models assume the Universe to be spatially homogeneous and isotropic. Isotropy, the equivalence of all directions from us, is on a fairly firm experimental basis provided by observations of the cosmic microwave background, the X-ray background and so on, although there are contradictory results (Brown, Mon.
Not. R. astr. Soc., 138, 527; 1968; Wilson, Mon. Not. R. astr. Soc. 155, 275; 1972) demanding further investigation. However, isotropy does not directly imply homogeneity, the equivalence of all points in space, for we might simply be at 'the centre of the Universe'. There is a strong anti-geocentric prejudice which favours homogeneity, but a lack of observational evidence.

The prejudice itself is recent. Only in 1918 did Shapley find the Sun was not at the centre of our Galaxy, three and a half centuries after Copernicus's discoveries. It was 1924 when Hubble proved some 'nebulae' were galaxies and 1952 when Baade's revision of the cosmic distance scale showed other galaxies could be as large as our own. The 'Copernican principle' and its strong form, the assumption of homogeneity, have subsequently been almost articles of faith, especially as they have the practical advantage of simplicity.

This attitude is reinforced by the difficulties of observationally checking homogeneity, arising because it involves a knowledge of conditions at all points, however distant. All one can hope to say is that any inhomogeneity has a scale greater than our range of observation. If the latter included our whole past we would know all we really require. But the finite velocity of light means we must compare distant objects as they were in the past and not as they are now, so we need to know about the evolution of both individual astrophysical objects and the Universe as a whole to make the comparison.

In spite of these difficulties, our view about homogeneity is naturally influenced by knowledge of the cosmic structure in our vicinity. The most obvious feature to test is the distribution of galaxies. It is now well known that galaxies are clustered and so not homogeneously distributed. de Vaucouleurs (for example in Science, 167, 1203; 1970) has expounded a theory of a hierarchy in which clusters are grouped in superclusters and so on. Peebles and collaborators (Astrophys. J., 196, 1; 1975 ) found no evidence of a hierarchy in their extensive statistical investigations of catalogues of galaxies. They concluded that there is a continuous spectrum of clusters on scales $10 / \mathrm{h}$ $\mathrm{kpc}$ to $5 / h \mathrm{Mpc}$, where $h$ represents the time cosmic distance scale in suitable units and $1 \mathrm{pc}$ is roughly $3 \times 10^{13} \mathrm{~km}$. Current estimates of $h$ are roughly in the range $\frac{1}{2}$ to 1 . This distribution could be the effect of gravitational attraction within initial accidental density enhancements, the clusters themselves being homogeneously distributed.

Turner and Gott (Astrophys. J., 197, L89; 1975) have just announced a new development. They were studying the problem of the non-luminous 'missing mass' that may be required to gravi- tationally bind clusters of galaxies, by examining the dynamics of small groups of galaxies. To find such groups they took all Northern Hemisphere galaxies brighter than fourteen magnitude (which corresponds to a distance of order roughly $100 \mathrm{Mpc}$ ) at more than forty degrees from the galactic plane, and separated these into two classes according to whether their nearest neighbour was at an angular separation greater or less than $45 \mathrm{~min}$ utes of arc. They noted that the more isolated class appeared to be homogeneously distributed in the sky, and thus tried applying Peebles's statistical methods to each class separately. They found the 'isolated' class, about $40 \%$ of the total, were indeed homogeneous. once effects of galactic obscuration and statistical noise had been allowed for. The other class however showed strong clustering on scales up to eight degrees.

This result, which invites further examination, will, if confirmed. leave us awkwardly poised. Each of the two classes separately is reasonably explicable, but Turner and Gott show from velocity and brightness data that they must be intermingled, and how one class became clumped while the other did not is a mystery. Once again nature proves to be more complex than was thought.

\section{Of bedsteads and neutrons}

from James Binney and Philip Candelas

INTEREST in the interaction of quantum theory and gravitation has been growing recently. Following Hawking's sensational prediction (Nature, 248, 30; 1974) that black holes may emit photons and other elementary particles, several recent publications have discussed the importance of quantum effects in strong gravitational fields (see review by DeWitt, Physics Reports, in the press; and Quantum Gravity: $A n$ Oxford Symposium, edit. by Isham, Penrose, and Sciama; Oxford University, 1975).

Just to make sure we have the basics right Colella, Overhauser and Werner (Phys. Rev. Lett., 34, 1472; 1975) have been checking up experimentally that the de Broglie waves associated with neutrons really are refracted by a gravitational field in the same way that proton wavepackets are refracted by an electric field. Colella and coworkers have cut from a single crystal of silicon a device which operates much as a Young's double slit experiment. Bragg diffraction in one part of the crystal splits a neutron beam into two, each being subsequently diffracted back towards the other by another part of the crystal. Colella et al. then monitor 